\title{
Application of Artificial Immune System in Constructing a Financial Early Warning System: An Example of Taiwanese Banking Industry
}

\author{
${\text { Jih-Chang } \text { Hsieh }^{1 *} \text {, Shih-Hsin Chen }}^{2}$, Pei-Chann Chang ${ }^{2,3}$ \\ ${ }^{1}$ Department of Finance, Vanung University, Chung-Li 32061, Tao-Yuan, Taiwan. \\ ${ }^{2}$ Department of Industrial Engineering and Management, Yuan-Ze University, Chung-Li, Tao- \\ Yuan, Taiwan. \\ ${ }^{3}$ Department of Information Management, Yuan-Ze University, Chung-Li, Tao-Yuan, Taiwan. \\ hjc@vnu.edu.tw, s939506@mail.yzu.edu.tw, iepchang@saturn.yzu.edu.tw
}

\begin{abstract}
In recent decades, soft computing techniques have broadly applied to solve complex problems. Among the soft computing techniques, Artificial Immune System (AIS) have appeared as a new approach dealing with classification problems. In this paper, an AIS algorithm is developed and applied to a two-group classification problem. An example of Taiwanese banking industry is discussed and the financial ratios of each bank from 1998 to 2002 were collected. This system has to distinguish the operational performance (good or bad) of each bank to offer a reference material for the managers or investors.

The performance of AIS is compared with other five early warning systems, namely, genetic neural networks (GNN), case-based reasoning (CBR), backpropagation neural network (BPN), logistic regression analysis $(L R)$, and quadratic discriminant analysis $(Q D A)$. The result indicates that the proposed AIS is over $10 \%$ better than the three soft computing early warning systems (GNN, CBR and BPN). The AIS outperforms the statistical early warning systems (LR and QDA) at least $24 \%$.
\end{abstract}

\section{Introduction}

The operation performance of each company can be represented by some management indices. Through understanding the changes among those indices, management staffs are able to make appropriate strategies. The companies today are very competitive in between. Therefore, to construct a financial early warning system must be meaningful in management.
Early research of financial early warning systems applied statistical methods such as discriminant analysis and logistic regression analysis. Cheng [4], Martin [12], Lane et al. [10], Sinkey [14], and Taffler [15] have applied Cox proportional hazards model and time varying covariates model, logit regression, Cox proportional hazards model, multivariate analysis, and discrimiant analysis to construct early warning systems respectively. In the recent, the statistical methods are replaced with soft computing techniques because soft computing techniques have been proven to be more effective and more efficient in a number of fields. Various soft computing techniques were ever considered on financial early warning systems such as Barniv et al. [1], Bell [2], Boritz and Kennedy [3], Colin [5], Etheridge and Sriram [6], Kingdom and Feldman [7], Lee et al. [11], and Odom and Sharda [13]. All the research showed satisfactory results. Freitas and Timmis [7] introduced the foundations of artificial immune systems for data mining. This paper discussed two issues in the data mining, namely, classification (supervised learning) and anomaly detection. The early warning system in this paper proposes to judge a company either good or bad. This is a two-group classification problem. Therefore an artificial immune system is applied to construct an early warning system for banking industry in Taiwan.

\section{Financial Early Warning System Based on Artificial Immune System}

The classification problem is solved by resource limited AIS (RLAIS) proposed by Timmis and Neal [16], which replaces the antibody of AIS (Timmis et al, [17]) by artificial recognition ball (ARB). The 
functionality of ARB is similar to the original antibody. However, the main difference is ARB that includes one to several antibodies it its repository. Therefore, it avoids all the antibodies connected to each other and the network grows exponentially. Because of this reason, the RLAIS works more efficient than the original version of AIS. In the infrastructure of RLAIS, a network affinity threshold (NAT) plays an important role which determines the data assignment and dictates when the ARBs to be connected in the network. When we input data to train the RLAIS, the algorithm selects a closest ARB for this data item if the distance between the ARB pattern and the data vector is less than the NAT. Otherwise, a new ARB is created and its pattern is set to the data vector. The formula of NAT is listed below:

\section{$\mathrm{NAT}=$}

$$
\sum_{i<j}^{n} \text { Euclidean }\left(\text { Data }_{i}, \text { Data }_{j}\right) / n * \sqrt{\text { features }}
$$
follows:

The pseudo code of the AIS algorithm is listed as

\author{
Algorithm: AIS_Main() \\ Iteration: The number of iterations of training \\ ROWS: The training data size \\ NAT: The network affinity threshold \\ trainingData: The training data \\ 1. Normalize(trainingData) \\ 2. Initialization() \\ 3. NAT $\leftarrow$ calcNAT () \\ 4. for $i=0$ to $n$ do \\ 5. for $\mathrm{j}=0$ to ROWS do \\ 6. $\quad$ distance $\leftarrow$ findClosestARB (trainingData[j]) \\ 7. if (distance < NAT) do \\ 8. allocate() \\ 9. $\quad \operatorname{cull}()$ \\ 10. else \\ 11. allocate() \\ 12. clone () \\ 13. $\quad \operatorname{cull}()$ \\ 14. End if \\ 15. End for \\ 16. End for
}

There are some parameters that are in the AIS algorithm, this study applied the configuration suggested by Timmis et al [17]. The settings are shown in Table 1.
Table 1. Hit ratios of early warning systems

\begin{tabular}{lc} 
Parameter & Settings \\
\hline Resources & 10 \\
Mortality & 1.0 \\
Decay & 0.99
\end{tabular}

\section{Case Study}

\subsection{Case statement}

The banking industry in Taiwan has a unique characteristic different from others all over the world. That is, Taiwanese banking industry is protected by government to prevent from severe financial crisis in order to stabilize the society. Government will takeover the problem bank in case of any out-of-control bank appears. There is no bankruptcy case in Taiwan. Therefore, simply two categorized performance of each bank (good or bad) is discussed in this paper.

Data of 49 banks are collected from 1998 to 2002. The data are not the latest because from 2002 on, banks in Taiwan are merged together as financial holding companies. Thus the before 2002 and after 2002 banking industry are different. Merges are still undergoing. It is not appropriate to collect data in such variable environment. Therefore the data before 2002 are used. The data include 28 input variables and 1 output variable. The input variables denote the financial ratios of the banks while the only output indicates a decision which a bank is judged to be good or bad.

\subsection{Early warning systems}

Hsieh et al. [8] integrated a genetic algorithm and neural network (GNN) to construct a financial early warning system for Taiwanese banking industry. The result was compared with four other early warning systems based on case-based reasoning (CBR), backpropagation neural network (BPN), logistic regression analysis (LR), and quadratic discriminant analysis (QDA) respectively. The comparison reported that GNN approach was with a superior performance. The result of Hsieh et al. [8] is applied and compared with AIS proposed in this research.

\subsection{Criterion for comparison}

Methods in this research are evaluated by hit ratio. If the output of each method is the same as the actual value, then it is classified as "Hit", else it is classified as "Miss" (like Table 2 shows). Therefore, hit ratio denotes the capability that each method makes correct decisions. A method with higher hit ratio represents an effective financial early warning system in this paper. 
Table 2. Hit or miss of the output

\begin{tabular}{|c|c|c|c|}
\hline \multicolumn{2}{|c|}{} & \multicolumn{2}{c|}{ Output } \\
\cline { 3 - 4 } \multicolumn{2}{|c|}{} & Good & Bad \\
\hline \multirow{2}{*}{$\begin{array}{c}\text { Actual } \\
\text { value }\end{array}$} & Good & Hit & Miss \\
\cline { 2 - 4 } & Bad & Miss & Hit \\
\hline
\end{tabular}

3.4 Numerical studies

The result reported in Table 3 indicates that the AIS in this research outperform other early warning systems based on other approaches. We had discovered that AIS is better than statistical early warning systems at least $24.85 \%$ while the AIS outperformed the GNN (the best performance among soft computing based approaches) by $11.59 \%$. The performance of other soft computing techniques (GNN, CBR, and BPN) is around $10 \%$ to $20 \%$ leading the statistical methods.

Table 3. Hit ratios of early warning systems

\begin{tabular}{cc}
\hline Systems & Hit Ratio \\
\hline AIS & $97.30 \%$ \\
\hline GNN & $85.71 \%$ \\
CBR & $84.62 \%$ \\
BPN & $80.39 \%$ \\
LR & $72.45 \%$ \\
QDA & $65.20 \%$ \\
\hline
\end{tabular}

\section{Conclusion}

AIS is applied to construct a financial early warning system for Taiwanese banking industry. Data collected from 1998 to 2002 are used to be a case study. AIS is compared with other five systems based on GNN, CBR, BPN, LR, and QDA respectively. The result has shown that AIS leads in all the comparisons. The gaps of AIS between the other approaches are significant. That means AIS is potential and can be further extended to the real-world situations.

\section{References}

[1] R. Barniv, A. Agarwal, and R. Leach, "Predicting the outcome following bankruptcy filing: A three-state classification using neural networks," Intelligent Systems in Accounting, Finance and Management, vol. 6, pp.177194, 1997.

[2] T. Bell, "Neural nets or the logit model? A comparison of each model's ability to predict commercial bank failures," Intelligent Systems in Accounting, Finance and Management, vol. 6, pp.249-264, 1997.

[3] J. Boritz and D. Kennedy, "Effectiveness of neural networks types for prediction of business failure," Expert Systems with Applications, vol. 9, pp.503-512, 1995.
[4] P. Y. K. Cheng, "Predicting bank failures: a comparison of the Cox proportional hazards model and the time varying covariates model" $\mathrm{PhD}$ Thesis. Nanyang Business School, Nanyang Technological University, Singapore, 2002.

[5] A. M. Colin, "Genetic algorithms for financial modeling. In G. J. Deboeck (Ed.)," Trading on the Edge, New York: Wiley, pp.148-173, 1994.

[6] H. Etheridge and R. Sriram, "A comparison of the relative costs of financial distress modles: Artificial neural networks, logit and multivariate discirminant analysis," Intelligent Systems in Accounting, Finance and Management, vol. 6, pp. 235-248, 1997.

[7] A. A. Freitas and J. Timmis, "Revisiting the Foundations of Artificial Immune Systems for Data Mining," Accepted by IEEE Transactions on Evolutionary Computation.

[8] J. C. Hsieh, P.C. Chang, and S. H. Chen, "Integration of Genetic Algorithm and Neural Network for Financial Early Warning System: An Example of Taiwanese Banking Industry," Proceedings of The First International Conference on Innovative Computing, Information and Control (ICICIC'06), Volume 1. Beijing, China, pp.562$565,2006$.

[9] J. Kingdom and K. Feldman, Genetic Algorithms for Bankruptcy Prediction, London: Search Space Research Report No. 01-95, Search Space Ltd, 1995.

[10] W. Lane, S. Looney, and J. Wansley, "An application of the Cox proportional hazards model to bank failure," Journal of Banking and Finance, vol. 10, pp.511-531, 1986.

[11] K. C. Lee, I. G., Han, and Y. Kwon, "Hybrid neural network models for bankruptcy predictions," Decision Support Systems, vol. 18, pp.63-72, 1996.

[12] D. Martin, "Early warning of bank failure: a logit regression approach," Journal of Banking and Finance, vol. 1. no. 3, pp.249-276, 1977.

[13] M. Odom and R. Sharda, "A neural networks model for bankruptcy prediction," Preceedings of the IEEE International Conference on Neural Network, vol. 2, pp.163-168, 1990.

[14] J. Jr. Sinkey, "A multivariate statistical analysis of the characteristics of problem banks," Journal of Finance, vol. 1, pp.21-36, 1975.

[15] R. Taffler, "Forecasting company failure in the UK using discriminant analysis and financial ratio data," Journal of Royal Statistics Society A, vol. 145, pp.342258, 1982.

[16] J. Timmis and M. Neal, "A resource limited artificial immune system for data analysis," Knowledge-Based Systems, 14(3-4), pp.121-130, 2001.

[17] J. Timmis, M. Neal, and J. Hunt, "An artificial immune system for data analysis," BioSystems, 55(1-3), pp.143$150,2000$. 